\title{
Relaciones sociedad-naturaleza y la pandemia del COVID-19: ¿vaso medio lleno o medio vacío?
}

\section{Nature-society Relations and the COVID-19 Pandemic: Glass Half Full or Half Empty?}

DOI $10.15517 /$ rr.v99i2.41923

\author{
Edgar Espinoza Cisneros ${ }^{1}$ \\ ${ }^{1}$ Escuela de Geografía, Universidad de Costa Rica, Costa Rica, edgar.espinoza@ucr.ac.cr
}

Fecha de recepción: 21 de mayo del 2020 Fecha de publicación: 4 de junio del 2020

\section{Resumen}

Las crisis, en muchos casos, impulsan cambios que marcan la evolución de las sociedades humanas. Esto ha aplicado en las relaciones sociedad-naturaleza, a través de un vínculo de realimentación donde las crisis determinan estas relaciones y viceversa. En este ensayo se reflexiona sobre algunas posibles avenidas de acción que se pueden tomar hacia sociedades más resilientes y sostenibles, motivadas por la pandemia del COVID-19. Además, se examina brevemente esa relación entre enfermedades infecciosas y acciones humanas sobre el ambiente, así como algunas iniciativas que se promueven actualmente para engendrar cambios sociales hacia la sostenibilidad a raíz de la crisis por la pandemia.

Palabras clave: Ambiente y sociedad, Bienestar humano, Cambio social, Crisis climática, Sostenibilidad.

\begin{abstract}
In many instances, crisis drive changes that mark societal evolution. This has applied to naturesociety relationships by the analysis whereby crises determine this type of relationships and viceversa. This essay examines some possible avenues of action, motivated by the COVID-19 pandemic, towards more resilient and sustainable societies. Furthermore, it briefly examines the relationship between infectious diseases and human environmental actions, as well as some initiatives that are being promoted to engender social changes towards sustainability.
\end{abstract}

Keywords: Climate crisis, Environment and society, Human wellbeing, Social change, Sustainability. 


\section{La pandemia del COVID-19 como el vaso}

La metáfora de «crisis como oportunidades» no podría tener mayor relevancia en la coyuntura actual por la pandemia del COVID-19. Ante las graves afectaciones sanitarias y socioeconómicas ocasionadas por esta pandemia en el mundo, muchos optimistas suelen ver el vaso medio lleno. Ese optimismo - esperanzador, pero a la vez frágil - lleva a ver un disturbio como una posible salvación. Del otro lado está el tentador pesimismo del vaso medio vacío. Es harto reconocido que las relaciones sociedad-naturaleza en nuestro mundo globalizado tienen ya sus décadas, incluso siglos, de ser insostenibles (MEA 2005), sin señas de un cambio esperanzador. La «Gran Aceleración» (Steffen et al. 2015), esa tendencia de degradación ambiental a gran escala desde mediados del siglo XX, no da señas de desacelerarse. Detrás de esta aceleración está un modelo económico que homologa el lucro con el bienestar, que ignora los límites ecológicos planetarios, y que exacerba disparidades sociales. Así, se ha consolidado una visión muy parcializada y peligrosa que pone a toda costa el enriquecimiento material por sobre el ambiente y el bienestar humano a mediano y largo plazo.

Desafortunadamente, estas presiones en los sistemas de vida planetarios van en aumento a pesar de esfuerzos de personas y grupos con sentido de compromiso, responsabilidad y sensibilidad. Esos esfuerzos, aunque loables, hasta la fecha han palidecido ante el embate de un (des)orden económico mundial que urge de un cambio drástico. ¿Hará falta, entonces, un disturbio como la actual pandemia para revertir esa tendencia insostenible? A lo largo de la historia, a raíz de grandes disturbios se han gestado cambios sociales que marcaron el rumbo de la humanidad, pero ¿funcionará ante los enormes retos contemporáneos, en un mundo tan globalizado, diverso y complejo?, ¿podrá esta pandemia catalizar un cambio social para bien? Más específicamente aplicado al actual contexto, en palabras del geógrafo Eric Lambin $(2012,54)$ : «iserá que la expansión mundial de enfermedades infecciosas emergentes tiene el potencial de bajarle el ritmo al crecimiento económico, moderar la tasa de evolución del sistema económico global, y estabilizarlo, de esta forma previniendo de que se salga de control y que tenga un efecto destructivo en la naturaleza?» (traducción propia).

\section{Relaciones sociedad-naturaleza y agentes patógenos}

Históricamente, las relaciones sociedad-naturaleza han estado detrás del surgimiento y la propagación de gran alcance de enfermedades infecciosas. Estas relaciones actúan como un bucle de realimentación donde, por un lado, la alteración ambiental influye en la incidencia de enfermedades infecciosas y, por otro, estas enfermedades determinan ciertas interacciones con el ambiente. Por ejemplo, el paso del nomadismo al sedentarismo en el Neolítico, hace 
aproximadamente 11.000 años, significó un contacto mucho más cercano entre humanos y animales, exacerbando las zoonosis (transmisión de enfermedades en animales a seres humanos). Además de este contacto, las prácticas agrícolas también favorecieron las enfermedades infecciosas a través de la irrigación o la fertilización del suelo con excrementos animales. El advenimiento de la agricultura también muy probablemente propició el origen de enfermedades como la malaria en África Occidental hace aproximadamente 4.000 años, a raíz de las modificaciones en el paisaje. Esa mayor incidencia de enfermedades, a su vez, obligó a un cambio en las prácticas agrícolas, en los métodos de producción y en las interacciones con animales.

Más adelante en el tiempo, en el milenio pasado, las rutas comerciales intercontinentales entre Asia y Europa, y luego entre Europa y América, propiciaron la difusión de enfermedades infecciosas originadas por relaciones humano-ambiente en sitios particulares. Muchas de esas enfermedades introducidas causaron estragos sin precedentes, como las millones de muertes por peste bubónica en Europa y por viruela, sarampión y tifus en América. En este último continente, estos cambios demográficos tuvieron como efecto colateral una recuperación considerable de gran parte de la cobertura forestal hacia el siglo XVII (Denevan 2005), lo que a su vez redujo las probabilidades de surgimiento o propagación de enfermedades.

Las alteraciones ambientales se exacerbarían considerablemente a partir del siglo XX, manifestada en la «Gran Aceleración». Estas presiones antropogénicas han comprometido significativamente la capacidad funcional de los sistemas de soporte de vida planetarios y han facilitado además un surgimiento y alcance de enfermedades infecciosas sin precedentes. El cambio climático antropogénico, el cambio de uso de la tierra, la intensificación de los sistemas agrícolas, el trasiego de especies silvestres, la producción animal industrial y la destrucción de la biodiversidad, entre otras acciones humanas, están llevando a esa mayor proclividad de enfermedades. Según la Organización Mundial de la Salud (OMS), desde 1940, por año, aparece en promedio una nueva enfermedad infecciosa. Los surgimientos de virus como el Ébola, del Nilo Occidental, de Nipah, y el Sars-Cov2, han estado cercanamente ligados con la degradación ambiental inducida por el ser humano. De hecho, los cambios ambientales aumentan el riesgo de aparición de un 83\% de las enfermedades más importantes registradas por la OMS (Lambin 2012).

Otros factores claramente inciden en que estas enfermedades tengan un mayor alcance, como por ejemplo las migraciones y la conectividad global por las redes de transporte y comercio. Muchas de estas enfermedades, en efecto, surgen en sitios donde confluyen altas densidades poblacionales, un comercio mal regulado de especies, y condiciones insalubres. En estos casos, suele haber un contacto cercano, frecuente y diverso entre humanos y animales. En Asia, por ejemplo, la producción animal para consumo humano se ha octuplicado en menos de 30 años. Concomitantemente, solo del sur de China han surgido cuatro pandemias en los últimos 150 años. 
Hoy, de todos los agentes patógenos que se conocen, entre un $60 \%$ y un $70 \%$ provienen de animales domesticados o silvestres.

En síntesis, los impactos ambientales de las actividades humanas a lo largo de la historia, pero especialmente en el último siglo, han comprometido el bienestar social a través de una mayor incidencia de enfermedades infecciosas. Ahora examinaremos brevemente la otra cara del bucle de realimentación: ¿podrían estas enfermedades infecciosas catalizar, para bien, un cambio permanente en las sociedades humanas?

\section{O la magnificación de lo de siempre, o el cambio}

A grandes rasgos, los escenarios sociedad-naturaleza pospandemia pueden ser dos. En el primero, el optimismo del vaso medio lleno, se aprovecharía la inercia de cambios provocada por la pandemia para consolidar modelos de desarrollo sostenibles y resilientes. En el segundo, el pesimismo del vaso medio vacío, se daría un efecto rebote donde más bien se redoblen las tendencias insostenibles «business-as-usual», con el propósito de reponer el «tiempo perdido». En este segundo escenario, las reducciones efímeras en algunos indicadores de degradación ambiental serían un mero efecto colateral de una crisis cuyas lecciones y potencial para engendrar un cambio se desaprovecharían. En esta sección, sin embargo, trataré la visión del vaso medio lleno.

El primer escenario inexorablemente implicaría, en primera instancia, adoptar paradigmas de desarrollo de crecimiento económico cero, de decrecimiento, o de un crecimiento «verde», todos los cuales de seguro traerían muchísima oposición. Sin embargo, desde hace ya algunas décadas, un creciente número de académicos y practicantes han estado impulsando enfoques cualitativos de desarrollo sustentados en el decrecimiento económico (Daly 1997), que trascienden conceptualizaciones de bienestar meramente pecuniarias. Aunque aún tenues, estos esfuerzos han ido dando sus frutos, por ejemplo, a través de una mayor inclusión de indicadores sociales y ambientales en los sistemas de contabilidad económica, así como en el diseño de marcos normativos que articulan mejor las diferentes concepciones de bienestar humano (Díaz et al. 2015). Estos esfuerzos se han fortalecido también con movimientos ciudadanos que claman por modelos de decrecimiento económico.

Esta transición hacia modelos económicos sostenibles indudablemente implicaría la institucionalización de paradigmas que vinculen estrechamente bienestar humano y naturaleza. La voracidad capitalista ha ignorado o, en el mejor de los casos, minimizado ese vínculo. Al hacerlo, sin embargo, se autodestruye el mismo aparato económico capitalista. En efecto, una de las grandes contradicciones del capitalismo es que tiende a agotar lo que necesita para funcionar (el capital natural). En su lugar debe surgir un paradigma que considere los límites de los sistemas de soporte 
de vida, y que reconozca y valore la amplia gama de servicios que nos brinda la naturaleza. Esto también conllevaría adoptar visiones de bienestar más multidimensionales, donde se contemplen distintos componentes. Por ejemplo, el confinamiento y el distanciamiento social pueden hacer que se valore más la recreación en la naturaleza o bien el fortalecimiento del sentido de comunidad en contextos naturales, lo que puede derivar en acciones más firmes para resguardar ecosistemas claves para nuestro bienestar. Esta pandemia puede entonces realzar la íntima conexión entre bienestar humano y naturaleza, y dejar más claro cómo nos afecta cuando la dañamos, y también cómo podría protegernos si la cuidamos.

Será clave también aprovechar la agudización de la sensibilidad social y ambiental que probablemente derivará de esta pandemia y canalizarla especialmente hacia el campo político y la movilización ciudadana para lograr acciones significativas para atender la crisis climática. El éxito de esto, no obstante, dependería de qué tanto se asocien con la crisis climática las afectaciones causadas por la pandemia, y lo que posiblemente podría suceder ante la inacción. Por ejemplo, percibir escenarios de crisis climática donde se saturen los servicios de salud, se den desabastecimientos de productos esenciales, se trastorne el flujo comercial, el transporte internacional y el sector turístico, se agraven conflictos geopolíticos y se pierdan millones de medios de vida alrededor del mundo, podría ayudar a que se haga ese vínculo. Así, los impactos sociales, económicos y ambientales de la pandemia podrían ser vistos como un preámbulo de lo que se avecinaría si no se toman medidas contundentes y prontas para atender el cambio climático. El objetivo es, entonces, que esa sensibilización agudizada nutra las voluntades políticas e individuales hacia la acción. Esto debe complementarse con un mayor protagonismo de las generaciones jóvenes como gestores de cambios al «business as usual». Muchos jóvenes alrededor del mundo están mostrando liderazgo, energía, interés y capacidad para participar activamente en la toma de decisiones y presionar por acciones más prontas y efectivas hacia un mundo mejor.

Sería fundamental también un reforzamiento de las políticas y regulaciones ambientales para garantizar la protección de ecosistemas claves y controlar la intrusión humana en áreas de alto valor ecológico. También, es de esperar que se implementen medidas para regular más las interacciones entre humanos y animales - ya sean domésticos o silvestres -, tengan o no trascendencia cultural. Esto implicaría controles más estrictos al manejo y venta de animales de consumo tanto domésticos como silvestres, así como también sanciones más rigurosas para el comercio de vida silvestre. Estas medidas, en principio, beneficiarían significativamente esfuerzos de conservación de la biodiversidad en muchas partes del mundo y con ello los beneficios que se obtienen de la naturaleza.

Es de esperar que los casos de desabastecimiento de ciertos productos en algunos países por la actual pandemia hagan que se tomen previsiones para garantizar una producción autosuficiente 
y sostenible que aumente la resiliencia a mediano y largo plazo, más aún ante los escenarios previstos por el cambio climático. Por eso, se puede avanzar sustancialmente en una revalorización de la soberanía y seguridad alimentaria, lo que implicaría repensar los métodos de producción, particularmente la intensificación productiva y su sostenibilidad en el tiempo. En su lugar se deberán impulsar medidas para mejorar la eficiencia productiva, cerrar brechas en el rendimiento, y el uso de la tecnología para procurar la sostenibilidad (Foley et al. 2013), por mencionar algunas. Pero, esto será positivo en tanto se mejoren los medios de vida rurales y se garantice la integridad ecológica de los sistemas productivos a mediano y largo plazo.

Como la sostenibilidad es inmanentemente social, esta crisis trae valiosas oportunidades para reducir disparidades sociales. En efecto, muchos de los problemas ambientales tienen como principal impulsor a la desigualdad social. En los últimos meses, en muchos países se han tomado medidas de asistencia social que podrían preceder el establecimiento de medidas estructurales permanentes hacia la reducción de las disparidades sociales. La pérdida de muchos empleos ha forzado a los gobiernos a tomar medidas para procurar el acceso a alimentos y servicios a sus poblaciones, así como en algunos casos promover contribuciones de trabajadores asalariados para financiar iniciativas de asistencia social. También ha habido movilizaciones ciudadanas para asistir a hogares con necesidad. Es clave, entonces, que estas medidas deriven en cambios permanentes como mejoras en el acceso a los servicios de salud, reforzamiento de la clase media, apoyo al acceso a la educación, reducción de las brechas salariales y eliminar privilegios desproporcionados en los sectores públicos, entre otras. Por otra parte, una mayor flexibilización en las jornadas de trabajo en particular y en las condiciones laborales en general podrían también derivar en reducciones sustanciales en el uso de combustibles fósiles, congestionamientos viales y contaminación del aire, acelerando el proceso de transición hacia una menor dependencia de esta fuente de energía.

Ya se están viendo iniciativas en el corto plazo para empezar a emprender este cambio. La Unión Europea, por ejemplo, recientemente lanzó una estrategia de biodiversidad para el 2030 motivada por la crisis de la pandemia (Comisión Europea 2020). La estrategia se apoya en la conciencia creada por esta crisis en cuanto al nexo entre salud humana y naturaleza y contempla, entre otras cosas, la recuperación de ecosistemas degradados, reducciones sustanciales en el uso de pesticidas, fomento más agresivo a las energías renovables, protección de especies polinizadoras, incentivos a la agricultura ecológica, y el resguardo de áreas marinas de alto valor ecológico. En Holanda, un grupo de cientos de académicos aprovechó la coyuntura del COVID19 para sugerir acciones hacia una pronta transición en ese país. Entre las sugerencias está el fomento de la inversión en sectores de la economía críticos para el bienestar público (como la educación, las energías renovables y la salud pública), la reducción sustancial en el consumo de combustibles fósiles y de materiales derivados de la minería, un viraje hacia una economía circular, 
el fomento a las economías agrarias locales junto con desincentivos a la producción ambientalmente dañina, y cambios a los patrones de consumo insostenibles. De manera similar, expertos de coaliciones científicas como la Plataforma intergubernamental Científico-Normativa sobre Diversidad Biológica y Servicios de los Ecosistemas (IPBES, por sus siglas en inglés) sugieren que los paquetes de estímulos de reactivación económica tengan como requisito el que las corporaciones beneficiarias se comprometan a realizar cambios urgentes y permanentes en su operación (Settele, Díaz, y Brondizio 2020). Sin embargo, será necesario esperar el apaciguamiento de las etapas críticas de la pandemia para diseñar paquetes de medidas que aligeren ese proceso de cambio hacia un mundo ambiental y socialmente sostenible.

\section{Conclusión}

¿Podrá la pandemia ser un disturbio que catalice un cambio social para bien? En este ensayo, se quiso reflexionar en torno a esta pregunta examinando posibles avenidas de acción, para bien, que podrían tomarse con la inercia de la crisis pandémica. Mucho del historial de las relaciones sociedad-naturaleza, en efecto, parece indicar que son los eventos abruptos y de impactos inmediatos y perceptibles (e.g. conflictos, pandemias, desastres naturales, etc.) los que más efectivamente suelen galvanizar cambios sociales para bien. Ante eventos más difusos, perentorios y mucho más complejos como el cambio climático, desafortunadamente como sociedad no se actúa con suficiente diligencia, por lo que aprovechar cualquier oportunidad para consolidar un cambio social para bien de una vez por todas es fundamental.

Para procurar estos cambios, es imprescindible sustituir el statu quo económico por modelos de desarrollo que internalicen la importancia de la naturaleza para el bienestar humano. Por eso, la inercia de esta actual crisis mundial debe canalizarse hacia este fin, extraer lecciones aprendidas y aplicarlas para atender los ingentes retos que tenemos por delante como humanidad. Al final, dependerá de nosotros como sociedad si se ve este evento como una oportunidad vacía y desperdiciada o un cambio lleno y esperanzador hacia la tan ansiada pero elusiva sostenibilidad. 


\section{Referencias}

Comisión Europea. 2020. "EU Biodiversity Strategy for 2030: Bringing nature back into our lives". Bruselas: Comisión https://ec.europa.eu/info/sites/info/files/communication-annex-eu-biodiversity-strategy2030_en.pdf

Daly, Herman. 1997. Beyond Growth: The Economics of Sustainable Development. Boston, MA: Beacon Press.

Denevan, William M. 2005. "The pristine myth: the landscape of the Americas in 1492". Annals of the Association of American Geographers 82 (3): 369-85.

Díaz, Sandra, Sebsebe Demissew, Julia Carabias, Carlos Joly, Mark Lonsdale, Neville Ash, Anne Larigauderie, et al. 2015. "The IPBES Conceptual Framework-connecting nature and people". Current Opinion in Environmental Sustainability 14: 1-16.

Foley, Jonathan A., Navin Ramankutty, Kate A. Brauman, Emily S. Cassidy, James S. Gerber, Matt Johnston, Nathaniel D. Mueller, et al. 2013. "Solutions for a cultivated planet". Nature, núm. 7468: 35.

Lambin, Eric. 2012. An Ecology of Happiness. Chicago: University of Chicago Press.

MEA, (Millennium Ecosystem Assessment). 2005. Ecosystems and Human Well-Being: Current State and Trends. Vol. 1. Millennium Ecosystem Assessment. Washington, D.C.: Island Press.

Settele, Josef, Sandra Díaz, y Eduardo Brondizio. 2020. “COVID-19 Stimulus Measures Must Save Lives, Protect Livelihoods, and Safeguard Nature to Reduce the Risk of Future Pandemics". Intergovernmental Science-Policy Platform on Biodiversity and Ecosystem Services (IPBES). 2020. https://ipbes.net/covid19stimulus.

Steffen, Will, Wendy Broadgate, Lisa Deutsch, Owen Gaffney, y Cornelia Ludwig. 2015. "The trajectory of the Anthropocene: the great acceleration". The Anthropocene Review 2 (1): $81-98$. 